\title{
Protective Effects of Aspirin from Cardiac Hypertrophy and Oxidative Stress in Cardiomyopathic Hamsters
}

\author{
Rong Wu, ${ }^{1}$ David Yin, ${ }^{1}$ Nataliya Sadekova, ${ }^{1}$ Christian F. Deschepper, ${ }^{2}$ Jacques de Champlain, ${ }^{1}$ \\ and Helene Girouard ${ }^{1}$ \\ ${ }^{1}$ Department of Pharmacology, Faculty of Medicine, Université de Montréal, Pavillon Roger-Gaudry, \\ 2900 Edouard Montpetit, Montréal QC, Canada H3T 1J4 \\ ${ }^{2}$ Clinical Research Institute of Montreal (CRIM), 110 Pine Ave. West, Montréal QC, Canada H2W $1 R 7$
}

Correspondence should be addressed to Helene Girouard, helene.girouard@umontreal.ca

Received 10 April 2012; Revised 31 May 2012; Accepted 1 June 2012

Academic Editor: Adrian Manea

Copyright (C) 2012 Rong Wu et al. This is an open access article distributed under the Creative Commons Attribution License, which permits unrestricted use, distribution, and reproduction in any medium, provided the original work is properly cited.

\begin{abstract}
Objective. To evaluate the capacity of chronic ASA therapy to prevent cardiac alterations and increased oxidative stress in cardiomyopathic hamsters. Methods and Results. Male Syrian cardiomyopathic and age-matched inbred control hamsters received ASA orally from the age of 60 days. Animals were sacrificed at the age of 150, 250, and 350 days to evaluate the time course of cardiac hypertrophy and cardiovascular tissue superoxide anion $\left(\mathrm{O}_{2}{ }^{-}\right)$production. At the age of 150 days, the ventricular weight over body weight ratio, resting heart rate, and cardiovascular $\mathrm{O}_{2}{ }^{-}$production were much higher in cardiomyopathic hamsters than those in control. At the age of 250 days, in addition to the continual deterioration of these parameters with age, the blood pressure started to fall and the signs of heart failure appeared. In these cardiomyopathic hamsters, chronic ASA treatment (a) completely prevented elevated $\mathrm{O}_{2}{ }^{-}$production and the $\mathrm{NAD}(\mathrm{P}) \mathrm{H}$ oxidase activity, (b) significantly slowed down the development of the cardiac hypertrophy and fibrosis. Conclusions. Chronic ASA treatment significantly prevents the deterioration of cardiac function and structure as well as the increased oxidative stress in the cardiomyopathic hamster. Our findings suggest that ASA presents a therapeutic potential to prevent cardiac dysfunction.
\end{abstract}

\section{Introduction}

The Syrian golden hamster of the cardiomyopathic strain is a model of hereditary cardiomyopathy that mimics the gradual progression of congestive heart failure in humans [1]. This animal model has a progressive nonpressure overload cardiac disease originated from a genetically transmitted metabolic anomaly that induces degenerative lesions in all striated muscles with particular consistency and intensity in the heart [2]. This type of cardiomyopathy is characterized by ventricular hypertrophy which progresses into dilated congestive heart failure in the later stages of the disease. The pathologic development can be divided into four temporal phases: prenecrotic (25-30 days), necrotic (70-75 days), hypertrophic (125-150 days) with progressing dilatation (225-250 days), and severe heart failure (325-350 days) [1].
The physiopathology of the disease state is not fully understood, but previous studies have shown an overactivated renin-angiotensin system which is characterized by higher plasma and ventricular angiotensin II concentrations [3], higher ventricular angiotensin converting enzyme (ACE) activity [4], and an upregulation of cardiac angiotensin II AT1 receptor in the cardiomyopathic hamsters [5]. Administration of ACE inhibitors such as quinapril [4] or captopril [6] preserves contractile function and/or increases survival rate in these animals.

Previous data suggest that the vascular renin-angiotensin system and the oxidative stress play a critical role in the generation of cardiomyopathies and heart failure [7]. Ang II has been shown to increase the cardiovascular tissue NAD $(\mathrm{P}) \mathrm{H}$ oxidase activity and to stimulate the cardiovascular $\mathrm{O}_{2}{ }^{-}$ production [8]. This oxidative mechanism is thought to play an important role in Ang-II-mediated trophic cardiovascular 
changes such as cardiovascular tissue hypertrophy and in hypertension [9].

Aspirin (acetylsalicylic acid (ASA)) is an anti-inflammatory and cardiovascular protective drug with potent inhibitory properties on cyclo-oxygenases which are responsible for the arachidonic acid metabolism and prostaglandin production. Although ASA has no direct effect on cardiovascular function, this drug has been reported to provide effective beneficial protection against many cardiovascular pathological conditions such as atherosclerosis, ischemic heart diseases, and myocardial infarction. Our previous studies have demonstrated that ASA is a potent antioxidative agent which markedly reduced the vascular $\mathrm{O}_{2}{ }^{-}$production by reducing the vascular $\mathrm{NAD}(\mathrm{P}) \mathrm{H}$ oxidase activity in normal and hypertensive rats [10]. Thus, our hypothesis is that ASA, by inhibiting $\mathrm{NAD}(\mathrm{P}) \mathrm{H}$ oxidase activity, prevents cardiovascular structural and functional alterations in the cardiomyopathic hamster.

The aims of this study are to evaluate the cardioprotective and antioxidant effects of chronic administration of ASA during the development of cardiac structural and functional alterations in the cardiomyopathic hamster.

\section{Methods}

2.1. Animals. Studies were performed on 36 male cardiomyopathic hamsters (CM, CHF 146) and an equivalent number of their age-matched male Syrian inbred control (Cntrl, CHF148) purchased from the Canadian Hybrid Farm (NS, Canada). The animals were housed individually in a temperature-controlled room with a 6:00 am to 6:00 pm light-dark cycle. Each type of animals was separated into placebo and ASA-treated groups. The ASA treatment started at the age of 60 days and continued until the end of the experiment (except when specified otherwise). The animals were given free access to drinking water. For the placebo groups, their drinking water was free of any drug, whereas aspirin was added in the drinking water of treated hamsters. The concentration of aspirin in drinking water was adjusted upon each animal's drinking volume to ensure a treatment dose of $100 \mathrm{mg} / \mathrm{kg} /$ day. Six hamsters from each group were sacrificed at the age of 150 days, 250 days and 350 days.

The blood pressure and heart rate were measured with the femoral artery cannulation method under general anaesthesia with a mixture of ketamine/xylazine $(90 / 5 \mathrm{mg} / \mathrm{kg}$ i.m.) just before the animal decapitation. The catheter was connected to a pressure transducer (MX860, Medex Inc. Carlsbad, CA) for continuous recording of arterial blood pressure and pulse rate for more than 10 mins via a patient monitor (model 206EL, Protocol Systems, Inc., Beaverton, OR). The heart and thoracic aorta were then quickly excised and immersed in ice-cold Krebs-Hepes buffer solution containing (mmol/L): $\mathrm{NaCl} 99.01, \mathrm{KCl} 4.69, \mathrm{CaCl} 2$ 1.87, MgSO 4 1.20, K2HPO4 1.03, NaHCO3 25.0, Na-Hepes 20.0, glucose 11.1 (saturated with $95 \% \mathrm{O}_{2}$ and $5 \% \mathrm{CO}_{2}$, $\mathrm{pH}$ 7.40). After the heart was washed and dissected, the ventricle was weighed and the aortic periadventitial tissue was carefully removed. The part of the cardiac apex was cut into $0.5 \mathrm{~mm}$ thick and roughly $10 \mathrm{mg}$ slices while the aorta was cut into $2 \mathrm{~mm}$ ring segments for $\mathrm{O}_{2}$ measurement. The rest of cardiac and aortic tissues were frozen in liquid nitrogen and kept at $-80^{\circ} \mathrm{C}$ until assayed.

In a preliminary study, eight 250 -day-old CM hamsters were treated with placebo or ASA $(100 \mathrm{mg} / \mathrm{kg} /$ day $)$ for 7 days. Compared with placebo-treated group, the ASA treatment did not modify any cardiovascular parameters (ventricle/body weight ratio, heart rate, or blood pressure). However a reduction of ventricular and aortic $\mathrm{O}_{2}{ }^{-}$production was observed (data not shown).

All experimental procedures were performed in accordance with the guidelines of the Canadian Council for Animal Care and monitored by an institutional care committee.

2.2. Superoxide Anion Measurement. The superoxide anion $\left(\mathrm{O}_{2}{ }^{-}\right)$production was measured using the lucigeninenhanced chemiluminescence method as described previously [11]. Briefly, after $10 \mathrm{~min}$ equilibration in KrebsHepes buffer at room temperature, the aortic ring or the myocardial slice was transferred to a scintillation vial containing $5 \mu \mathrm{mol} / \mathrm{L}$ lucigenin to determine the basal $\mathrm{O}_{2}{ }^{-}$ level. The chemiluminescence was recorded every minute for 15 minutes by a liquid scintillation counter (Wallac 1409, Turku, Finland) switched to the out-of-coincidence mode. At the end of the count, the fresh cardiac tissue and aortic ring were weighed. The data were expressed as cpm per mg tissue.

To evaluate the intrinsic $\mathrm{NAD}(\mathrm{P}) \mathrm{H}$ oxidase activity, an $\mathrm{NAD}(\mathrm{P}) \mathrm{H}$ oxidase inhibitor, diphenyleneiodonium (DPI, $100 \mu \mathrm{mol} / \mathrm{L}$ ) was used to determine DPI-inhibitable production of $\mathrm{O}_{2}{ }^{-}$. Aortic rings or cardiac slices were incubated first with DPI for $10 \mathrm{~min}$ at room temperature before $\mathrm{O}_{2}{ }^{-}$ production was evaluated. DPI-inhibitable production of $\mathrm{O}_{2}{ }^{-}$was calculated as the difference in production of $\mathrm{O}_{2}{ }^{-}$ observed in the presence and in the absence of DPI.

2.3. NAD $(P) H$ Oxidase Subunit p47phox Western Blot Analysis. Frozen ventricular tissue was crushed in liquid nitrogen. Lyses and Western blots were performed as described previously [12] with $20 \mu \mathrm{g}$ of proteins loaded on gels. Proteins were separated (Mini Gel Protean II System, Bio-Rad) and then transferred electrophoretically to nitrocellulose paper at $70 \mathrm{~V}$ for $90 \mathrm{~min}$. After transfer, the membranes were washed twice and then incubated in PBS containing 5\% dehydrated milk at room temperature for $2 \mathrm{~h}$. The blots were then incubated with specific antibody against p47phox (H195, sc14015, Santa Cruz Biotechnology) with a 1/2000 dilution. The antibody-antigen complexes were detected by second antibody (goat anti-rabbit IgG-HRP, 1: 4000, Santa Cruz Biotechnology). Membranes were exposed to Kodak X-Omat blue films (Kodak, Rochester, NY, USA) for $2 \mathrm{~min}$. The gels were scanned and the densitometry of the bands was assessed using Scion image 4.0.2 (Scion Corporation, Frederick, MD, USA). Results for each condition were divided by the values of normal inbred hamsters without aspirin treatment to estimate the relative magnitude of changes. The $\beta$-actin $(42 \mathrm{kDa})$ was used as our loading control for p47phox 
expression experiments. All reagents used in buffer solution were purchased from Sigma Chemical Co.

2.4. Ventricular Fibrosis. Hearts were fixed in formalin for $24 \mathrm{~h}$. Two cross-sections of the heart were obtained at $1-\mathrm{mm}$ intervals midway between the base and the apex of the heart. Ventricular fibrosis was determined from the tissues obtained from the two cross sections. These tissues were dehydrated and embedded in paraffin, and $4 \mu \mathrm{m}$ sections were cut. A representative section from the two cross-sections of each heart was stained with picrosirius red. These sections were mounted on a slide for projection to a magnification of 20x. A digital image of the entire section was obtained using computer software (Aperio Technologies, CA, USA). The extent of fibrosis in the myocardium was assessed using the positive pixel count algorithm as the area of collagen staining which was expressed as a percentage of the total myocardial tissue area.

2.5. Data Analysis. Data are expressed as mean \pm SEM. Statistical comparisons were made by one-way ANOVA followed by Tukey test for multivariance. The critical level of significance was set at $P<0.05$.

\section{Results}

3.1. Body Weight and Cardiac Hypertrophy. Compared with inbred control hamsters, the body weight of the CM hamsters was significantly lower in all age groups (Figure $1(\mathrm{a})$ ). The body weight of both strains (control and CM hamsters) remained stable during the study period and was not modified by ASA treatment.

Cardiac hypertrophy was evaluated by measuring the ratio of cardiac ventricular weight over body weight at the ages of 150, 250, and 350 days (Figure 1(b)). The ventricle/body weight ratio remained low and stable in the inbred control animals during the whole study period. This ratio progressively increased with age in CM hamsters from the initial value of $3.41 \pm 0.06$ at 150 days to $3.63 \pm 0.01$ at 250 days and $4.07 \pm 0.10$ at 350 days $(P<0.01$ each compared with its previous data). ASA treatment reduced this ventricle/body weight ratio since age of 150 days and then partially prevented the increase of this ratio with age in CM hamsters (Figure 1(b)). ASA treatment did not show any effect on the ventricle/body weight ratio in inbred control hamsters.

3.2. Heart Rate and Blood Pressure. Heart rate (HR) was stable in inbred control hamsters during the whole study period (HR varied from 199 to 214 with an average of $205 \pm 5$ beat/min), while the HR was higher in CM hamster than the one of their age-matched inbred control animals since the age of 150 days $(210 \pm 6 \mathrm{beat} / \mathrm{min})$ and reached to even a higher level at age of 350 days $(241 \pm 4$ beat $/ \mathrm{min})(P<0.01$ versus Control) (Figure 2(a)). ASA treatment slowed down significantly HR increase in CM hamsters. Nevertheless, administration of ASA did not show any modification of the $\mathrm{HR}$ in the inbred control animals.
The systolic (SBP) and diastolic (DBP) blood pressure were similar between control and CM hamsters at the age of 150 days (control 123/82 versus CM 120/79 mmHg). Thereafter, these pressures continually decreased with age to a SBP of $110 \pm 5$ and $82 \pm 3 \mathrm{mmHg}$ and DBP of $75 \pm 3$ and $56 \pm$ $4 \mathrm{mmHg}$ in 250- and 350-day-old CM hamsters, respectively, which were significantly lower than those of age-matched control $(P<0.01)$. Meanwhile, the blood pressures remained stable in inbred control animals. ASA treatment significantly prevented blood pressure lowering in CM hamsters without modifying blood pressures in age-matched inbred control hamsters (Figure 2(b)).

Starting at the age of 250 days, the CM hamsters began to show some signs of heart failure such as reduced food intake, oedema, and ascites (data not shown).

3.3. Ventricular Fibrosis. The extent of fibrosis in ventricular tissues was assessed in 350-day-old control and CM hamsters treated either with placebo or ASA. The percentage of the tissue occupied by fibrosis was much higher in CM group $(16.5 \pm 1.4 \%)$ when compared with the control $(5.1 \pm 0.8 \%$, $P<0.01$, Figure 3). This high level of fibrosis in CM hamsters was reduced by $35 \%(P<0.01$ versus placebo-treated $\mathrm{CM})$ after ASA treatment, but no change was observed in ASAtreated control group.

3.4. Ventricular and Aortic Superoxide Anion Production. The cardiac ventricular and aortic tissue $\mathrm{O}_{2}{ }^{-}$production was evaluated in inbred control and $\mathrm{CM}$ hamster at the ages of 150, 250, and 350 days. Ventricular $\mathrm{O}_{2}{ }^{-}$production was higher in $\mathrm{CM}$ hamster than in their age-matched control. This high level of $\mathrm{O}_{2}{ }^{-}$production increased continually with age in CM group from a basal level of $243 \pm 16 \mathrm{cpm} / \mathrm{mg}$ tissue at the age of 150 days to $299 \pm 19 \mathrm{cpm} / \mathrm{mg}$ tissue at the age of 350 days, while the $\mathrm{O}_{2}{ }^{-}$level remained low and stable in the control group (Figure 4(a)). ASA treatment normalized the elevated ventricular $\mathrm{O}_{2}{ }^{-}$production in all age groups of CM hamsters $(P<0.01$ versus age-matched placebo-treated $\mathrm{CM}$ ), but only slightly reduced the $\mathrm{O}_{2}{ }^{-}$production in all age groups of inbred control animals.

Similar results were observed about the aortic $\mathrm{O}_{2}{ }^{-}$ production (Figure 4(b)). The aortic $\mathrm{O}_{2}{ }^{-}$production was $727 \pm 55,749 \pm 17$, and $741 \pm 38 \mathrm{cpm} / \mathrm{mg}$ tissue in $150-, 250$ and 350-day-old control animals, respectively. Compared with the controls, the aortic $\mathrm{O}_{2}{ }^{-}$production was elevated by $20 \%, 26 \%$, and $31 \%(P<0.01)$ in the age-matched $\mathrm{CM}$ hamsters. ASA treatment completely normalized this elevated $\mathrm{O}_{2}{ }^{-}$production level to $692 \pm 28,742 \pm 38$, and $755 \pm$ $29 \mathrm{cpm} / \mathrm{mg}$ tissue in the 3 age groups of CM hamsters $(P<$ 0.01 versus placebo-treated $\mathrm{CM}$ ). However, ASA treatment did not show significant effect in the inbred control groups.

3.5. NAD $(P) H$ Oxidase Activity and Expression of Cardiac $N A D(P) H$ Oxidase Subunit p47phox. The NAD $(\mathrm{P}) \mathrm{H}$ oxidase activity was estimated by DPI-inhibitable production of $\mathrm{O}_{2}{ }^{-}$anion in ventricular and aortic tissues from 350-dayold control and CM hamsters. The DPI-inhibitable $\mathrm{O}_{2}{ }^{-}$ production was significantly increased in ventricular and 


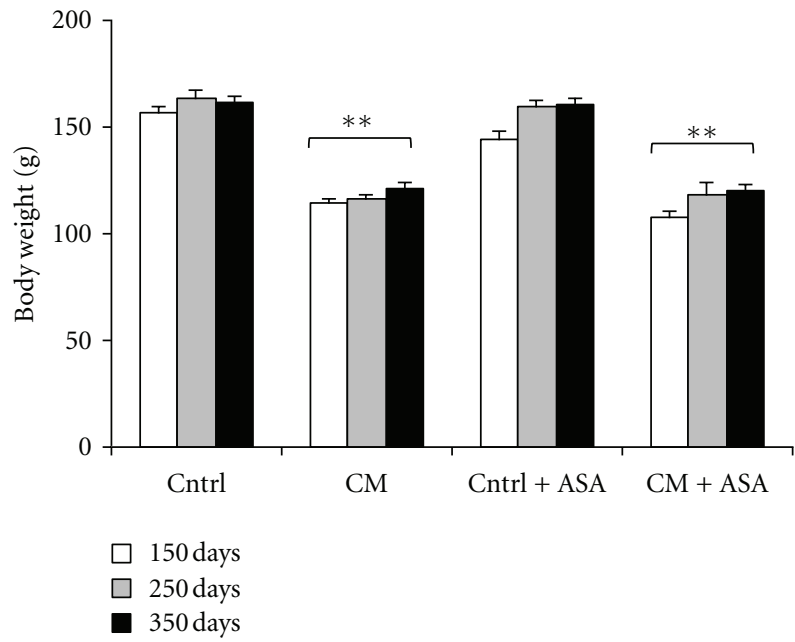

(a)

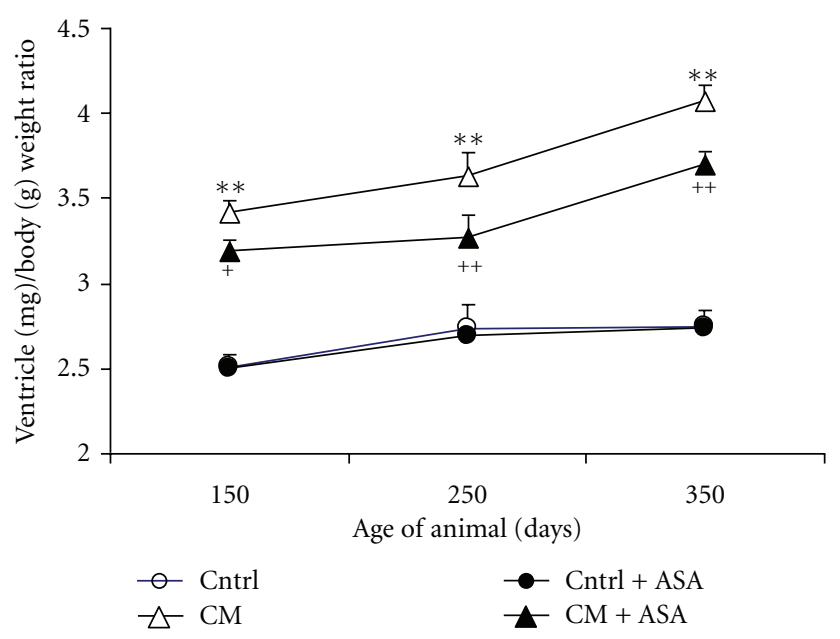

(b)

Figure 1: Time course of body weight (a) and the cardiac ventricular weight (mg)/body weight (g) ratio (b) in control (Cntrl) and cardiomyopathic $(\mathrm{CM})$ hamster treated with placebo or $100 \mathrm{mg} / \mathrm{kg} /$ day acetylsalicylic acid $(+\mathrm{ASA}) .{ }^{* *} P<0.01$ versus control, ${ }^{+} P<0.05$, ${ }^{++} P<0.01$ versus $\mathrm{CM}$ hamsters.

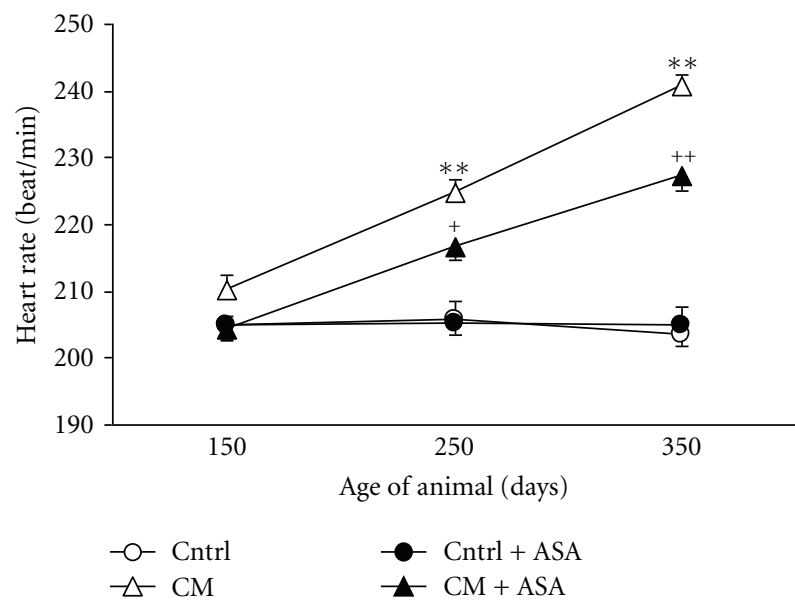

(a)

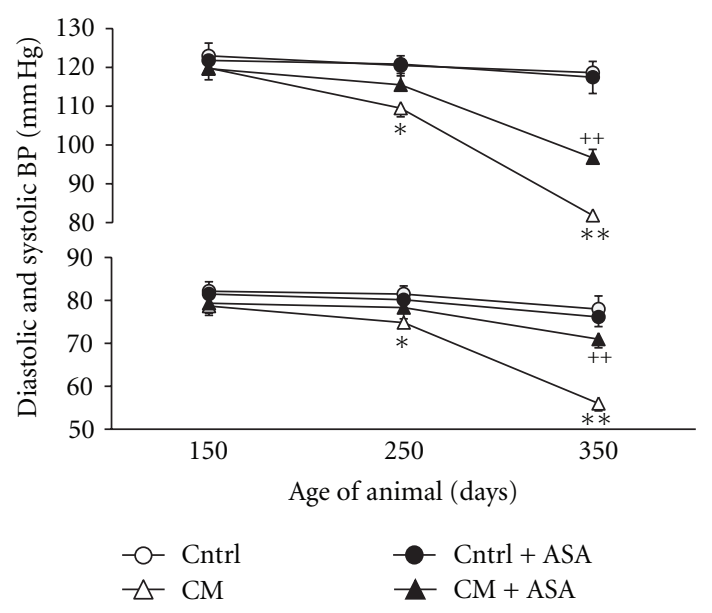

(b)

FIGURE 2: Selected hemodynamic parameters at the ages of 150, 250, and 350 days. (a) Heart rate in control (Cntrl) and cardiomyopathic (CM) hamsters with (+ASA) or without ASA treatment. (b) Systolic and diastolic blood pressure in the same animals. ${ }^{*} P<0.05,{ }^{* *} P<0.01$ versus control, ${ }^{++} P<0.01$ versus placebo-treated CM hamsters.

aortic tissues of CM hamsters by 47 and 32\% compared to the normal values of $130 \pm 14$ and $497 \pm 25 \mathrm{cpm} / \mathrm{mg}$ tissue obtained in the same tissues from inbred control animals (Figures 5(a) and 5(b)). ASA treatment normalized these elevated ventricular and aortic DPI-inhibitable productions of $\mathrm{O}_{2}{ }^{-}$in $\mathrm{CM}$ hamsters, but did not affect the $\mathrm{O}_{2}{ }^{-}$ production in inbred control animals.

The expression of cardiac tissue $\mathrm{NAD}(\mathrm{P}) \mathrm{H}$ oxidase subunit p47 was measured in 350-day-old control and CM hamsters by Western blot method. The ratio of the expression of p47phox in CM over their inbred controls was of $1.18 \pm$ 0.04 in $\mathrm{CM}$ group $(P<0.01)$. ASA treatment reduced this elevated ratio of expression to near control level $(P<0.05)$ (Figure 6).

\section{Discussion}

In the present study, the cardiomyopathy progressed with age in the CM hamsters. At the age of 150 days, the cardiac function was still in a compensatory stage presenting only cardiac hypertrophy and tachycardia. Thereafter, the SBP and DBP started to fall with age and signs of heart failure such as reduction of food intake, oedema, and ascites began to appear. These age-related cardiac damages are consistent with previous reports $[1,13]$. Meanwhile, the cardiovascular tissue $\mathrm{O}_{2}{ }^{-}$production was significantly elevated in the CM hamsters with an increased $\operatorname{NAD}(\mathrm{P}) \mathrm{H}$ oxidase activity and expression of the subunit p47phox of $\mathrm{NAD}(\mathrm{P}) \mathrm{H}$ oxidase. 

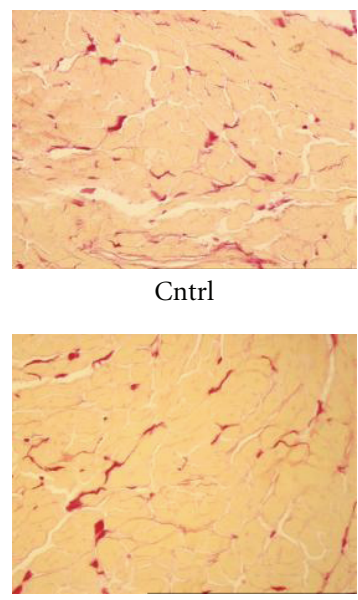

Cntrl + ASA

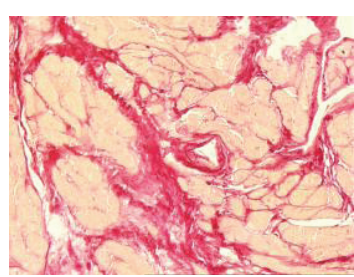

$\mathrm{CM}$

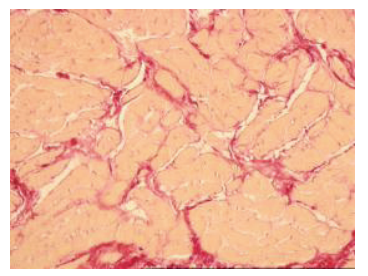

$\mathrm{CM}+\mathrm{ASA}$

(a)

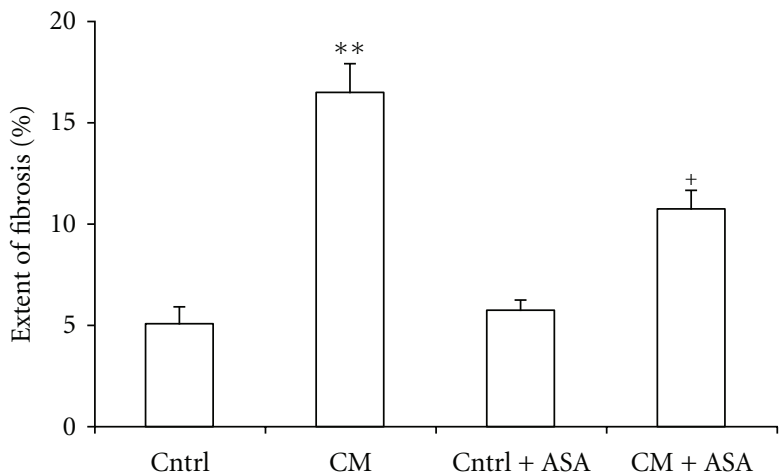

(b)

Figure 3: (a) Representative cross-sectional tissue slices of hearts stained with picrosirius red show minimal fibrosis in control (Cntrl) and importantly increased fibrosis in 350-day-old cardiomyopathic hearts (CM). ASA treatment significantly reduced the cardiomyotic fibrosis in the CM hamsters (CM + ASA), but did not show any effect on control (Cntrl + ASA), (b) Percentage of the tissue occupied by fibrosis in whole ventricular section of 350-day-old untreated or ASA-treated control or cardiomyopathic hamsters. ${ }^{* *} P<0.01$ versus control, ${ }^{+} P<0.05$ versus $\mathrm{CM}$ hamsters.

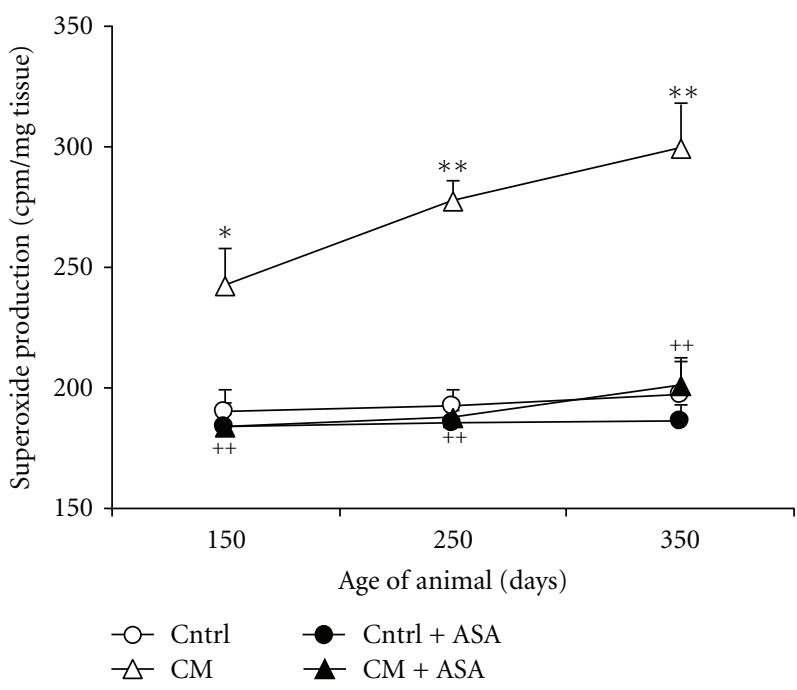

(a)

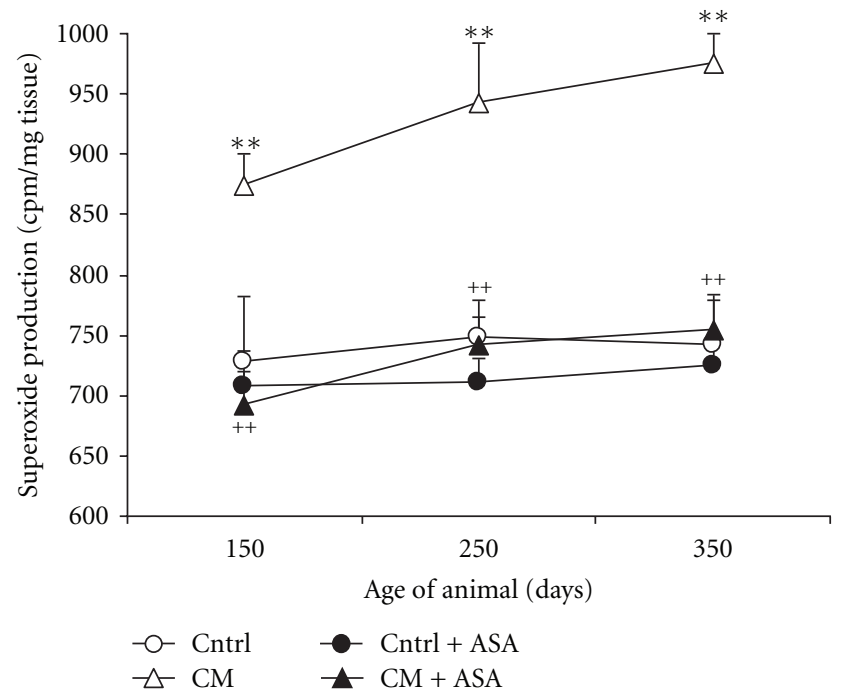

(b)

Figure 4: Time course of ventricular (a) and aortic (b) production of $\mathrm{O}_{2}{ }^{-}$in control (Cntrl) and cardiomyopathic (CM) hamsters with (+ASA) or without acetylsalicylic acid treatment. ${ }^{*} P<0.05,{ }^{* *} P<0.01$ versus control, ${ }^{++} P<0.01 \mathrm{CM}+\mathrm{ASA}$ versus CM hamsters.

An important finding from the present study is that chronic ASA treatment significantly slowed down the development of cardiac hypertrophy by reducing ventricle/body weight ratio and the extent of ventricular fibrosis in Syrian CM hamster. Moreover, ASA treatment prevented $\mathrm{HR}$ increase as well as blood pressure decrease suggesting improvement of both chronotropic and inotropic cardiac functions. Consistent with our previous report [10], ASA treatment demonstrated a potent antioxidative effect in the present animal model by completely normalizing the elevated cardiac and aortic tissue $\mathrm{O}_{2}{ }^{-}$production in $\mathrm{CM}$ hamsters. The DPI-inhibitable $\mathrm{O}_{2}{ }^{-}$production and the expression of the subunit p47phox of $\mathrm{NAD}(\mathrm{P}) \mathrm{H}$ oxidase were both reduced by ASA treatment suggesting that the antioxidative effect of ASA is probably mainly mediated by reduction of the $\mathrm{NAD}(\mathrm{P}) \mathrm{H}$ oxidase activity and expression. Our results also indicated that the DPI-inhibitable $\mathrm{O}_{2}{ }^{-}$production significantly contributes to the total cardiovascular tissue $\mathrm{O}_{2}{ }^{-}$production. Nevertheless, DPI is not a specific inhibitor of NADPH oxidase as it could also inhibit the NO synthase and cytochrome P450. These two enzymes could potentially modify the level of superoxide and other 


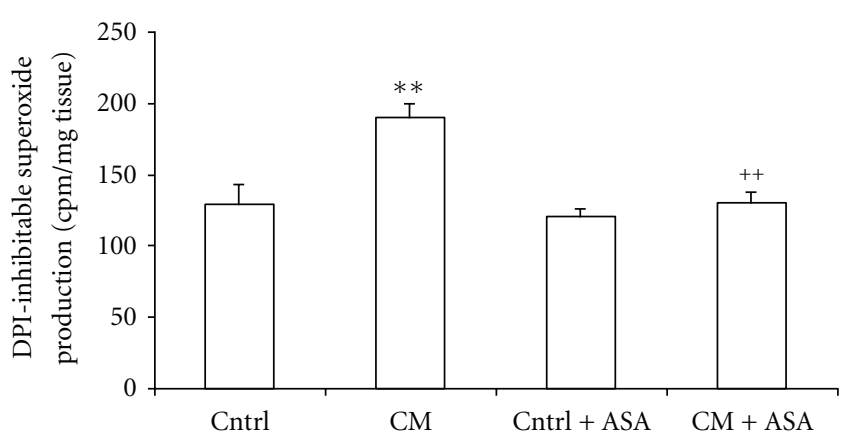

(a)

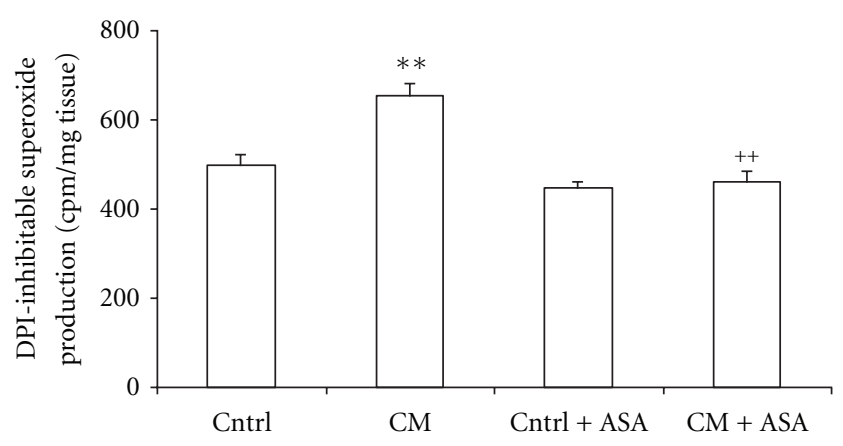

(b)

FIGURE 5: Diphenyleneiodonium (DPI)-inhibitable $\mathrm{O}_{2}{ }^{-}$production in ventricular slices (a) or aortic rings (b) from control (Cntrl) and cardiomyopathic (CM) hamsters treated with placebo or acetylsalicylic acid (+ASA). ${ }^{* *} P<0.01$ versus control, ${ }^{++} P<0.01$ versus $\mathrm{CM}$ hamsters.

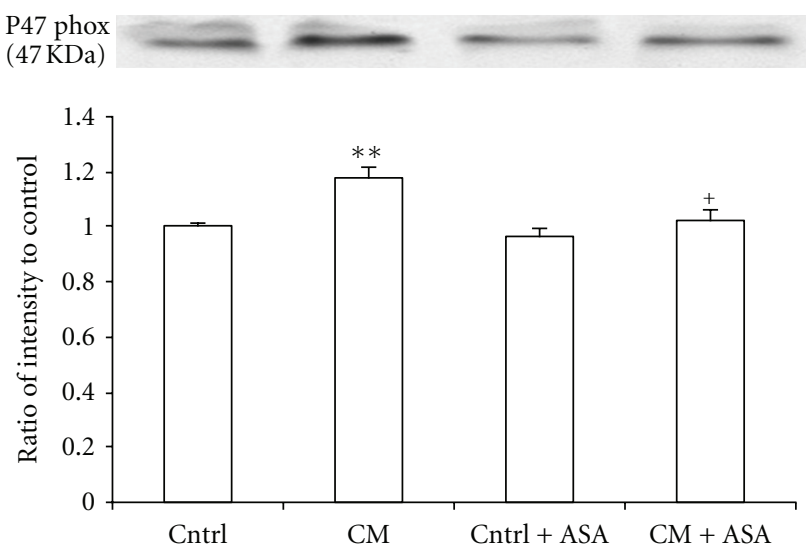

FIgure 6: The ratio of cardiac tissue $\mathrm{NAD}(\mathrm{P}) \mathrm{H}$ oxidase subunit p47phox expression over their control determined by Western blot in cardiomyopathic (CM) hamsters treated with placebo or $100 \mathrm{mg} / \mathrm{kg} /$ day acetylsalicylic acid (+ASA). ${ }^{* *} P<0.01$ versus control, ${ }^{+} P<0.05$ versus $\mathrm{CM}$ hamsters.

reactive oxygen species by changing the NO level or on the P450-related metabolism. These nonspecific effects of DPI could result in an imprecise estimation of the contribution of NADPH oxidase to the oxidative stress with the use of the DPI-inhibitable superoxide production only. However, the increased expression of p47phox strongly suggests an increased NADPH oxidase activity.

The pathophysiology of the cardiomyopathy in the Syrian hamster appears to be multifactorial [7]. Overactivation of the renin-angiotensin II system has been reported in this CM model, in which a high level of angiotensin converting enzyme activity and AT1 receptors in the cardiovascular tissues was observed at an early age $[4,5]$. These upregulations could lead to dysfunction of coronary vasculature, promoting vasospasms and transient ischemia. The latter was assumed to lead the early phase of myocardial necrosis and myolysis [14]. Increasing evidence indicates that activation of angiotensin II system can increase cardiovascular tissue $\mathrm{O}_{2}{ }^{-}$production and oxidative stress through the $\mathrm{NAD}(\mathrm{P}) \mathrm{H}$ oxidase pathway $[8,15]$. It is well known that oxidative-stress-mediated cardiac injury is implicated in the pathophysiology and development of cardiomyopathy and heart failure through inducing cellular injury and apoptosis, depressing myocardial contractility, and damaging coronary endothelial function, and myocardial blood supply $[7,16]$.

ASA has been reported to provide effective beneficial protection against many cardiovascular pathological conditions, such as atherosclerosis, ischemic heart diseases, and myocardial infarction. Our previous studies have demonstrated that ASA is a potent antioxidative agent that remarkably reduced $\mathrm{O}_{2}{ }^{-}$production in cardiovascular tissues and prevented the development of hypertension, vascular hypertrophy, insulin resistance in numerous pathologic conditions including SHR, chronically glucose-fed rats, and Ang II infusion-induced hypertension models [17, 18]. Moreover, ASA treatment abolished chronic Ang II infusion-induced oxidative stress, cardiac hypertrophy and hypertension in rats. It is worth noticing that ASA does not have any effect on cardiac functions such as contractility, heart rate and hypertrophy in healthy animals. Indeed, acute ASA administration did not show any effect on cardiovascular function or $\mathrm{O}_{2}{ }^{-}$production [10]. Our previous studies have demonstrated that all those beneficial cardiovascular effects of ASA are preventive and require long-term treatment. In the present study, short term ASA treatment (1 week) did not ameliorate cardiovascular functions or reduce cardiac hypertrophy in CM hamsters. Based on all these elements, we presume that the protective cardiovascular effects of ASA on the CM hamsters are indirect and probably mediated by its antioxidative properties. Our previous results also indicated that the antioxidative and cardiovascular protective properties of ASA were probably mediated by its effect on COX-2 and were not shared by other anti-inflammatory drugs with non-selective COX inhibitory property such as ibuprofen, indomethacin or salicylic acid [19].

In the present study, only early started (at age of 60 days) long-term ASA treatment slowed down the cardiac hypertrophic development and partially prevented the deterioration of the cardiac function. During this early age, the pathology changes in CM hamsters are characterised by coronary spasm, cardiac micronecrosis, and absence of any 
sign of cardiac function damage [20]. At that moment, the antioxidative property of ASA could protect and improve the endothelial function, reduce the vasospasm and cardiac ischemia, and prevent the micronecrosis, which slow down the cardiac hypertrophy and heart failure development.

The pathophysiology of the cardiomyopathy in the Syrian hamster is not well understood. Our results demonstrate that long-term ASA treatment can slow down the deterioration of cardiac functions suggesting that oxidative stress might play an important role in the development of heart failure in this CM model. However, although ASA treatment normalizes the cardiovascular $\mathrm{O}_{2}{ }^{-}$production and NADPH oxidase activity, it can only partially prevent the deterioration of the cardiac functions. These results suggest that some oxidative stress-independent mechanism might be involved in the disease development.

It is worth to notice that the body weight of CM hamsters was significantly lower than that of control animal in the present study. This could result in an overestimation of the cardiac hypertrophy in CM hamsters. However, the ventricle/body weight ratios clearly demonstrate the development of hypertrophy over time as well as the protective effect of ASA which does not have any effect on body weight.

In conclusion, the long-term ASA treatment significantly prevents cardiac structure and function alterations in the $\mathrm{CM}$ hamster. It seems that the preventive effects of ASA are mediated by its antioxidative properties. Thus, our findings suggest a potentially beneficial therapeutic use of ASA in treatment of heart failure.

\section{Acknowledgments}

The authors would like to express their gratitude to Diane Papin and Sylvie Picard for their technical expertise as well as to Dr. Chantal Lambert for her helpful comments. This study was supported by funds from the Canadian Institutes in Health Research (CIHR) grants to J. Champlain and H. Girouard and the Canada Foundation for Innovation (CFI) to H. Girouard. J. Champlain was the holder of a J. C. Edwards career investigatorship. H. Girouard was the holder of an operating grants from the Fonds de Recherche en Santé du Québec (FRSQ), Natural Science and Engineering Research Council of Canada (NSERC), and a new investigator award from the FRSQ and the HSFC.

\section{References}

[1] E. G. Hunter, V. Hughes, and J. White, "Cardiomyopathic hamsters, CHF 146 and CHF 147: a preliminary study," Canadian Journal of Physiology and Pharmacology, vol. 62, no. 11, pp. 1423-1428, 1984.

[2] D. Chemla, E. Scalbert, P. Desche, and Y. Lecarpentier, "Cardiomyopathy in the Syrian hamster. Physiological and therapeutic aspects," Archives des Maladies du Coeur et des Vaisseaux, vol. 84, no. 4, pp. 85-87, 1991.

[3] F. Nakamura, M. Nagano, R. Kobayashi et al., "Chronic administration of angiotensin II receptor antagonist, TCV116 , in cardiomyopathic hamsters," American Journal of Physiology, vol. 267, no. 6, pp. H2297-H2304, 1994.
[4] S. J. Haleen, R. E. Weishaar, R. W. Overhiser et al., "Effects of quinapril, a new angiotensin converting enzyme inhibitor, on left ventricular failure and survival in the cardiomyopathic hamster. Hemodynamic, morphological, and biochemical correlates," Circulation Research, vol. 68, no. 5, pp. 1302-1312, 1991.

[5] C. Lambert, Y. Massillon, and S. Meloche, "Upregulation of cardiac angiotensin II AT1 receptors in congenital cardiomyopathic hamsters," Circulation Research, vol. 77, no. 5, pp. 10011007, 1995.

[6] H. Hirakata, F. M. Fouad-Tarazi, F. M. Bumpus et al., "Angiotensins and the failing heart. Enhanced positive inotropic response to angiotensin I in cardiomyopathic hamster heart in the presence of captopril," Circulation Research, vol. 66, no. 4, pp. 891-899, 1990.

[7] N. Escobales and M. J. Crespo, "Early pathophysiological alterations in experimental cardiomyopathy: the Syrian cardiomyopathic hamster," Puerto Rico Health Sciences Journal, vol. 27, no. 4, pp. 307-314, 2008.

[8] K. K. Griendling, C. A. Minieri, J. D. Ollerenshaw, and R. W. Alexander, "Angiotensin II stimulates NADH and NADPH oxidase activity in cultured vascular smooth muscle cells," Circulation Research, vol. 74, no. 6, pp. 1141-1148, 1994.

[9] J. F. Reckelhoff and J. C. Romero, "Role of oxidative stress in angiotensin-induced hypertension," American Journal of Physiology, vol. 284, no. 4, pp. R893-R912, 2003.

[10] R. Wu, D. Lamontagne, and J. de Champlain, "Antioxidative properties of acetylsalicylic acid on vascular tissues from normotensive and spontaneously hypertensive rats," Circulation, vol. 105, no. 3, pp. 387-392, 2002.

[11] T. Munzel, H. Sayegh, B. A. Freeman, M. M. Tarpey, and D. G. Harrison, "Evidence for enhanced vascular superoxide anion production in nitrate tolerance. A novel mechanism underlying tolerance and cross-tolerance," Journal of Clinical Investigation, vol. 95, no. 1, pp. 187-194, 1995.

[12] K. Matrougui, Y. E. Eskildsen-Helmond, A. Fiebeler et al., "Angiotensin II stimulates extracellular signal-regulated kinase activity in intact pressurized rat mesenteric resistance arteries," Hypertension, vol. 36, no. 4, pp. 617-621, 2000.

[13] N. R. Bastien, A. V. Juneau, J. Ouellette, and C. Lambert, "Chronic AT1 receptor blockade and angiotensin-converting enzyme (ACE) inhibition in (CHF 146) cardiomyopathic hamsters: effects on cardiac hypertrophy and survival," Cardiovascular Research, vol. 43, no. 1, pp. 77-85, 1999.

[14] S. M. Factor, T. Minase, S. Cho, R. Dominitz, and E. H. Sonnenblick, "Microvascular spasm in the cardiomyopathic Syrian hamster: a preventable cause of focal myocardial necrosis," Circulation, vol. 66, no. 2, pp. 342-354, 1982.

[15] H. Hitomi, H. Kiyomoto, and A. Nishiyama, "Angiotensin II and oxidative stress," Current Opinion in Cardiology, vol. 22, no. 4, pp. 311-315, 2007.

[16] M. J. Thomson, M. P. Frenneaux, and J. C. Kaski, "Antioxidant treatment for heart failure: friend or foe?" QJM, vol. 102, no. 5, pp. 305-310, 2009.

[17] A. El Midaoui, R. Wu, and J. de Champlain, "Prevention of hypertension, hyperglycemia and vascular oxidative stress by aspirin treatment in chronically glucose-fed rats," Journal of Hypertension, vol. 20, no. 7, pp. 1407-1412, 2002.

[18] R. Wu, M. A. Laplante, and J. de Champlain, "Prevention of angiotensin II-induced hypertension, cardiovascular hypertrophy and oxidative stress by acetylsalicylic acid in rats," Journal of Hypertension, vol. 22, no. 4, pp. 793-801, 2004.

[19] R. Wu, M. A. Laplante, and J. de Champlain, "Cyclooxygenase2 inhibitors attenuate angiotensin II-induced oxidative stress, 
hypertension, and cardiac hypertrophy in rats," Hypertension, vol. 45, no. 6, pp. 1139-1144, 2005.

[20] N. Escobales and M. J. Crespo, "Angiotensin II-dependent vascular alterations in young cardiomyopathic hamsters: role for oxidative stress," Vascular Pharmacology, vol. 44, no. 1, pp. 22-28, 2006. 


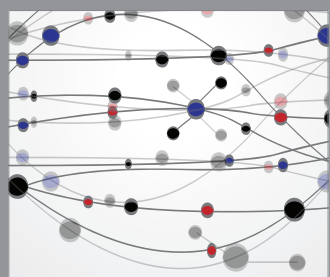

The Scientific World Journal
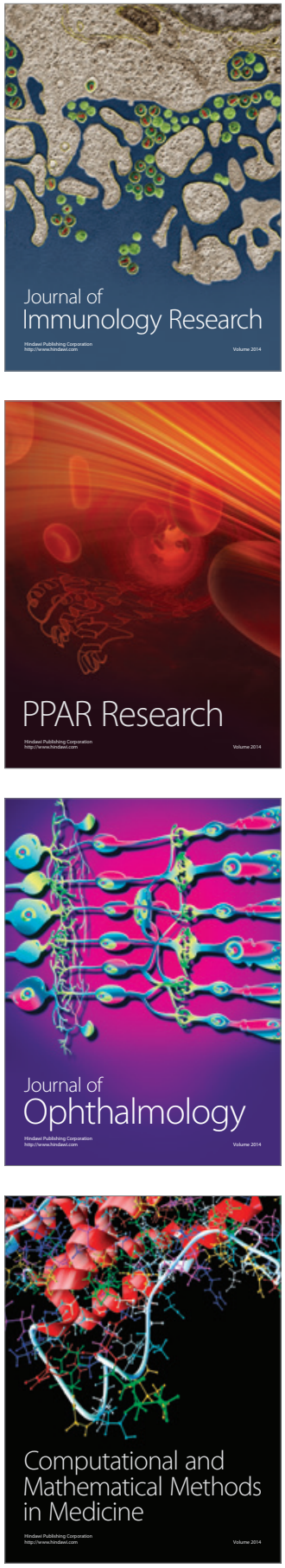

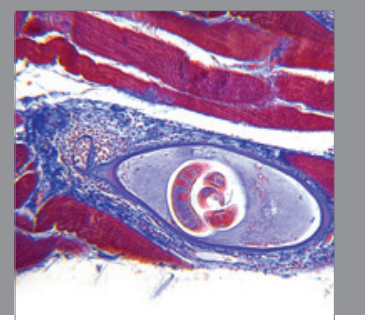

Gastroenterology

Research and Practice
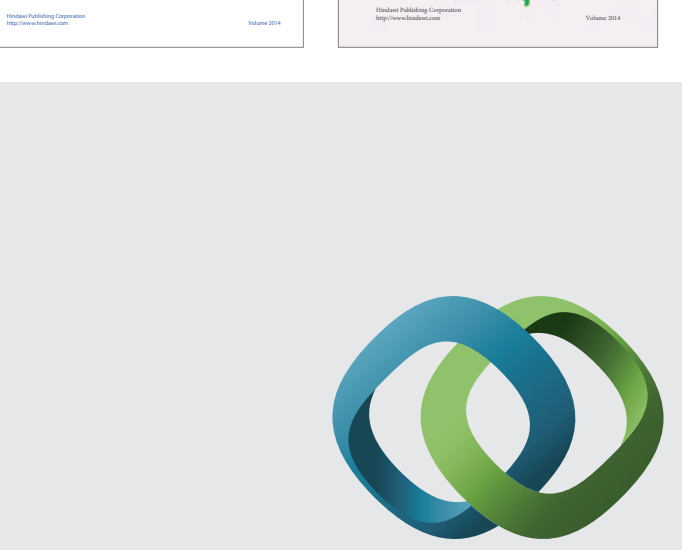

\section{Hindawi}

Submit your manuscripts at

http://www.hindawi.com
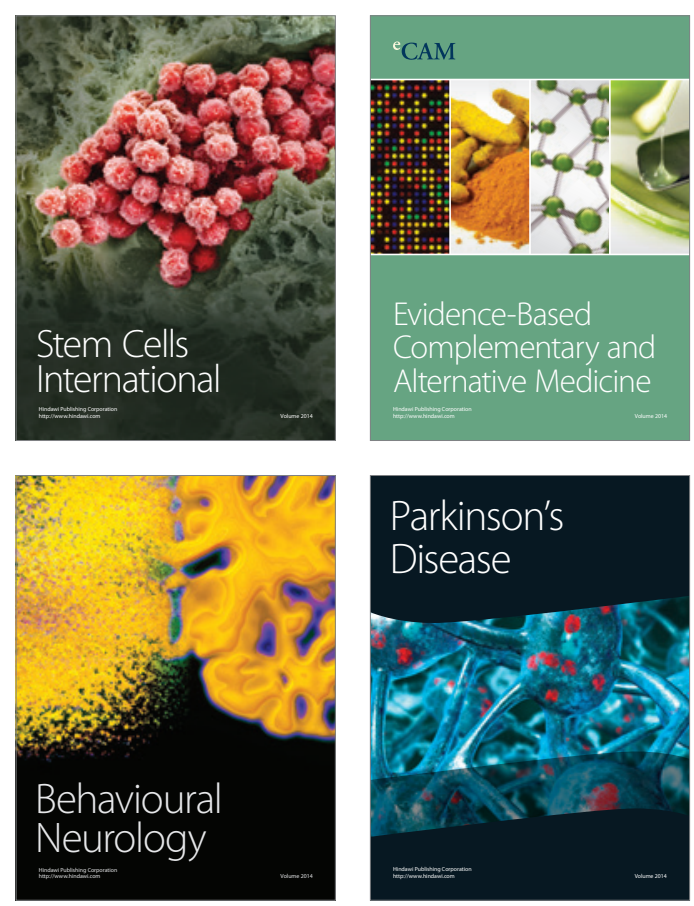

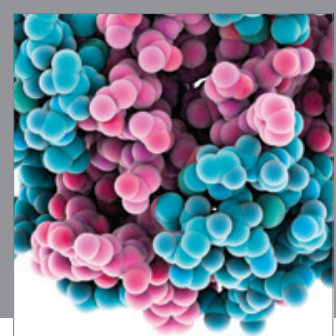

Journal of
Diabetes Research

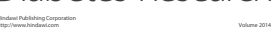

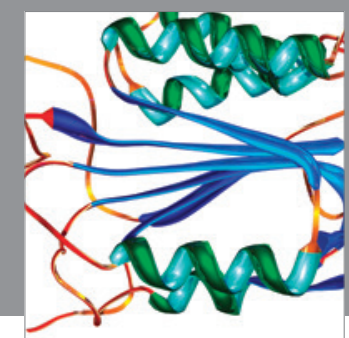

Disease Markers
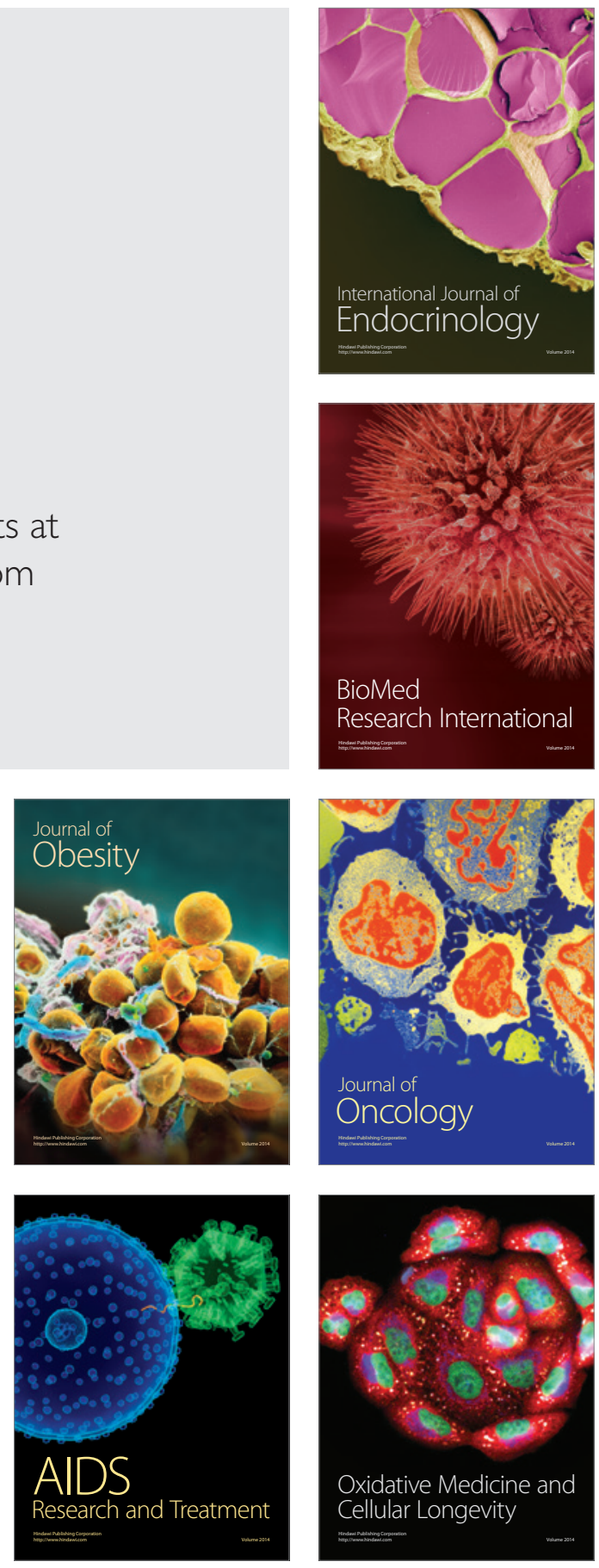